\title{
INTRINSIC MICROWAVE PHASE NOISE OF FIBER-OPTIC LINKS
}

\section{TH3C-3}

\author{
Paul J. Matthews and Ronald D. Esman \\ Naval Research Laboratory \\ Code 5672, Optical Sciences Division \\ Washington, DC 20375-5338
}

\begin{abstract}
Measurements of the residual, singlesideband microwave phase noise added by a fiber-optic link are presented. The measurements are made without the use of RF amplifiers and therefore show the true residual phase noise of the link for the first time. Measurements of $1.3 \mu \mathrm{m}$ and $1.55 \mu \mathrm{m}$, externally-modulated links indicate a very low residual phase noise.
\end{abstract}

\section{INTRODUCTION}

Fiber-optic links possess many advantages over various other transmission media such as coaxial cable; including low intrinsic loss, increased immunity to electro-magnetic interference, reduced weight and the potential for signal processing. Due to these and other advantages, analog fiber-optic links are being incorporated into systems for microwave signal transmission and processing at a rapidly increasing rate. Many of the current and future applications for fiber-optic microwave systems have very stringent system phase noise requirements. Examples include highperformance radar systems and the distribution of precise frequency and timing standards for radio astronomy. There have been numerous attempts to measure the residual phase noise of various fiber-optic links [1-5]. Due to the difficulties involved in making accurate phase noise measurements, these links have all included some form of electronic postamplification to decrease the system measurement noise floor to acceptable levels. The use of microwave amplifiers makes it difficult to ascertain the true phase noise of the fiber-optic link. Also, only a handful of these

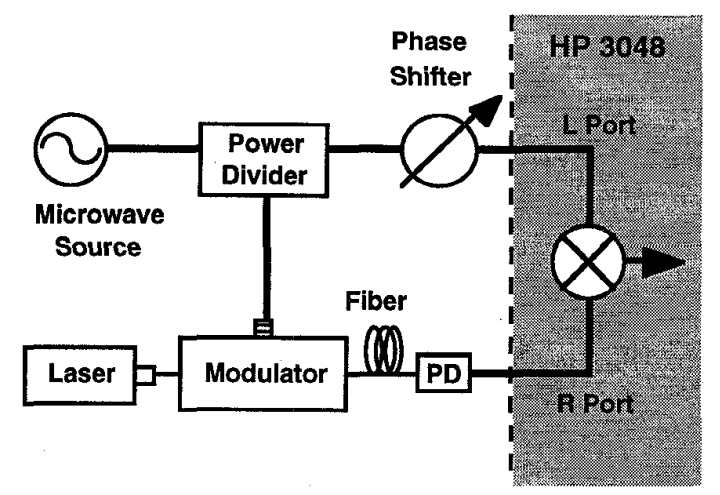

Figure 1. Externally modulated fiber-optic link and single-sideband phase noise measurement set-up.

measurements were performed at microwave frequencies.

In this paper, the first measurements of the true residual, single-sideband (SSB) phase noise of $1.3 \mu \mathrm{m}$ and $1.5 \mu \mathrm{m}$, externally-modulated fiber-optic links at microwave frequencies are presented. A low system noise floor is obtained without incorporating microwave amplifiers into the link through the use of high power photodetectors. Results using this technique indicate excellent phase noise performance with an Allan variance of $3.1 \times 10^{-26}$ for a 0.1 second measurement time ( $40 \mathrm{MHz}$ bandwidth).

\section{SYSTEM CONFIGURATION}

The fiber-optic link and measurement set-up are depicted in Figure 1. A standard residual phase noise measurement using a phase detector is employed with the fiber-optic link feeding one port of the mixer in the phase noise measurement system (Hewlett-Packard 3048). A low-phase noise microwave source was amplified and split with a $3 \mathrm{~dB}$ coupler to serve 


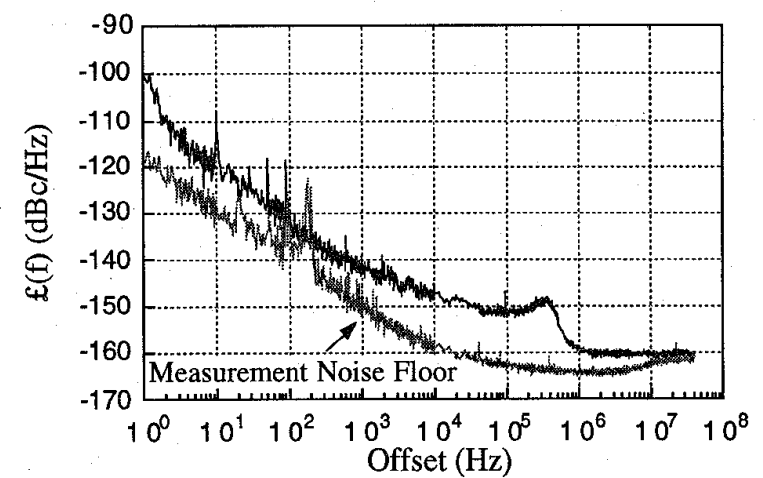

Figure 2. The SSB phase noise of the $1.3 \mu \mathrm{m}$ fiber-optic link at $10.2 \mathrm{GHz}$ along with the measured noise floor. The output RF power of the link was 0 $\mathrm{dBm}$.

as the input signal for the fiber-optic link and the reference signal for the phase noise measurement. The fiber-optic link consisted of an optical source, a dual-output, Mach-Zehnder modulator and a high-power $\mathrm{p}-\mathrm{i}-\mathrm{n}$ diode photodetector. Two optical sources were used a $285 \mathrm{~mW}, 1.3 \mu \mathrm{m}$ solid-state laser and a 35 $\mathrm{mW}, 1.5 \mu \mathrm{m}$ distributed feedback (DFB) laser. The photodetectors were capable of handling currents of approximately $20 \mathrm{~mA}$, resulting in a microwave power sufficient to drive the input port of the phase noise measurement system without microwave amplification.

\section{RESULTS AND DISCUSSION}

The measured SSB phase noise at a microwave frequency of $10.2 \mathrm{GHz}$ for the 1.3

$\mu \mathrm{m}$ link is shown in Figure 2 along with the measured system noise floor. The link delivered a microwave power of $0 \mathrm{dBm}$ to the phase noise test set and contained approximately 9 meters of SMF-28 fiber. The delay due to the fiber was equalized in the reference arm to within $0.2 \mathrm{~ns}$ using a length of coaxial cable. An excellent phase noise of $-147 \mathrm{dBc} / \mathrm{Hz}$ at an offset frequency of $10 \mathrm{kHz}$ is obtained. Beyond about $1 \mathrm{kHz}$, there is a distinct plateau in the data as a

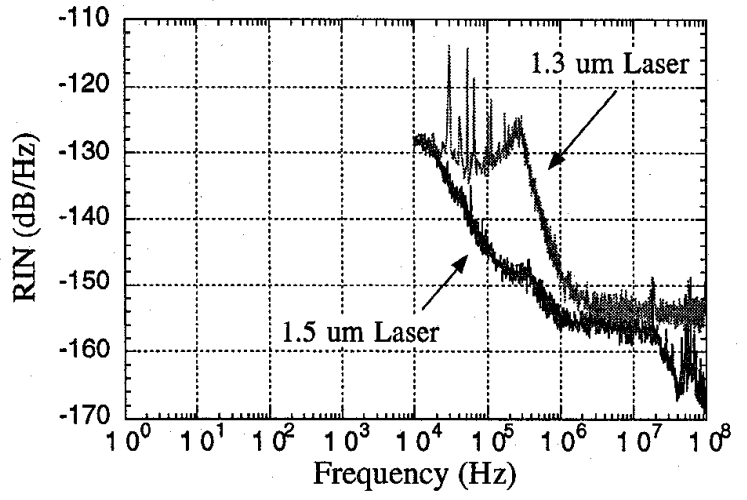

Figure 3. The measured RIN of the $1.3 \mu \mathrm{m}$ and $1.5 \mu \mathrm{m}$ lasers.

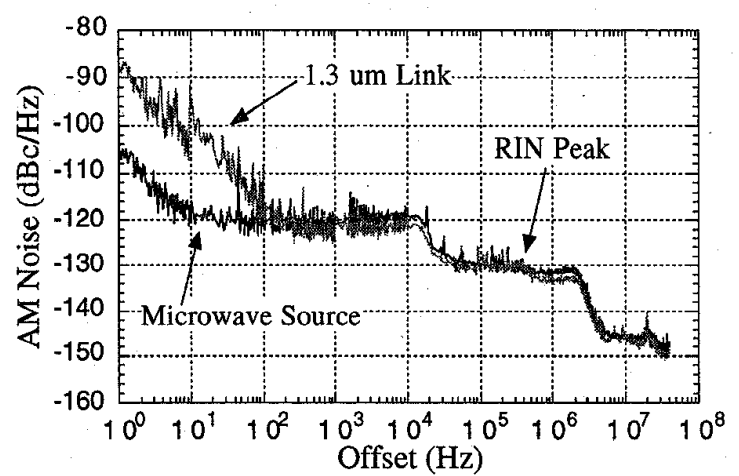

Figure 4. The measured AM noise of the 1.3 $\mu \mathrm{m}$ link along with the AM noise of the microwave source.

result of the laser RIN. The laser relaxation oscillation at $\sim 300 \mathrm{kHz}$ is readily evident. The measured RIN for the laser is shown in Figure 3 and exhibits the same features present in Figure 2. The Allan variance was calculated from the data in Figure 2 to be $3.1 \times 10^{-26}$ for a 0.1 second measurement time and a $40 \mathrm{MHz}$ bandwidth.

In a typical residual phase noise measurement, the balanced mixer has only 20 to $30 \mathrm{~dB}$ of $\mathrm{AM}$ noise rejection. Thus, the AM noise of the system should be evaluated in order to detect any AM leak-through that may appear in the phase noise measurement. The measured AM noise of the link is shown in Figure 4 as is the AM noise of the microwave source. The RIN peak is just visible in the data and is at a 


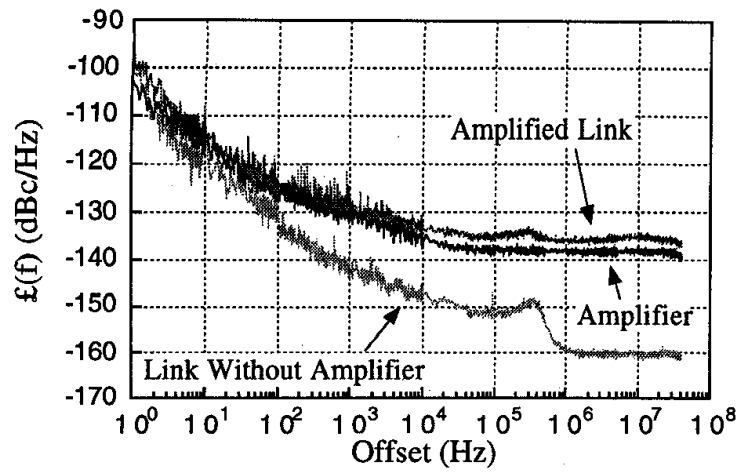

Figure 5. The SSB phase noise of the $1.3 \mu \mathrm{m}$ fiber-optic link with and without microwave amplification. The SSB phase noise of the amplifier is also shown.

level where it may effect the phase noise measurements. Since there is no common-mode rejection of $A M$ noise in a residual phase noise measurement, the measured AM noise of the link also includes the AM noise of the microwave source. Due to equipment limitations at the time of these measurements, the microwave source used in the phase noise measurements is not the same as the one used for the AM noise measurement. The source used for the AM measurements was somewhat noisier than that used for the phase noise measurements, especially at the lower offset frequencies. However, it is apparent that the phase noise of the link, shown in Figure 2, exhibits some AM leak-through due to the high AM noise of the link. Thus, the actual intrinsic phase noise of the link may be lower.

The SSB phase noise of the link with a lownoise microwave post-amplifier was also measured and the results shown in Figure 5. It is readily apparent that inclusion of the microwave amplifier in the measurement has raised the measured phase noise and completely obscured the prominent features from the laser RIN seen in the previous measurement. This is due to the low power input to the microwave amplifier which sets the noise floor for large offsets through the noise figure of the amplifier. The noise floor of the amplified link was estimated

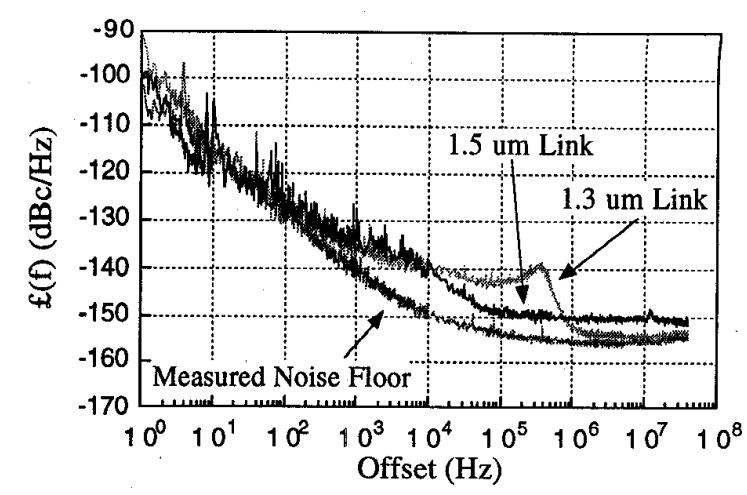

Figure 6. The SSB phase noise of the $1.5 \mu \mathrm{m}$ fiber-optic link at $10.2 \mathrm{GHz}$ along with the measured noise floor. The output RF power of the link was -10 $\mathrm{dBm}$.

to be $-138 \mathrm{dBc} / \mathrm{Hz}$, very close to the measured value of $-136 \mathrm{dBc} / \mathrm{Hz}$.

The SSB phase noise for the $1.5 \mu \mathrm{m}$ link is shown in Figure 6 for an output RF power of $10.0 \mathrm{dBm}$. When compared to the $1.3 \mu \mathrm{m}$ link at a similar RF output power, lower results are obtained from $10 \mathrm{kHz}$ to $700 \mathrm{kHz}$ due to the lower RIN of the $1.5 \mu \mathrm{m}$ DFB laser (Figure 3). For offsets below $200 \mathrm{~Hz}$, the results are at the measurement noise floor due to a lower output power. The Allan variance for this link was 2.7 $x 10^{-25}$ ( 0.1 second measurement time, $40 \mathrm{MHz}$ bandwidth).

\section{CONCLUSION}

We have measured for the first time the true residual, SSB phase noise added by a fiber-optic link to an analog microwave signal. Since no microwave amplification is used in the link, the results are more accurate and much lower than previous SSB phase noise measurements on fiber links have indicated. Both $1.3 \mu \mathrm{m}$ and 1.5 $\mu \mathrm{m}$, externally-modulated links are measured. An Allan variance of $3.1 \times 10^{-26}$ is obtained for the $1.5 \mu \mathrm{m}$ fiber-optic link with a 0.1 second measurement time and a $40 \mathrm{MHz}$ bandwidth. 


\section{ACKNOWLEDGMENTS}

This work was supported by the Office of Naval Research.

\section{REFERENCES}

[1]. I. Newberg, C. Gee, G. Thurmond and H. Yen, "Fiber optic delay lines for radar applications," Proc. SPIE, vol. 996, pp. 130-134, 1988.

[2]. R. Logan and G. Lutes, "High stability fiber optic systems: demonstrations and applications," Proc. 1992 IEEE Freq. Control Symposium, pp. 310-316, 1996.
[3]. C. Sun. G. Anderson, R. Orazi, M. Berry, M. Shadaram and S. Pappert, "Phase and amplitude stability of broadband analog fiber optic links," Proc. SPIE, vol. 2560, pp. 50-56, 1995.

[4]. T. Lane and J. Scheer, "Fiber optic link phase noise and drift effects on bistatic imaging radar performance," Proc. IEEE 1996 National Aerospace and Electron. Conf., Dayton, Ohio, pp. 648-654, May 2023, 1996.

[5]. P. Matthews, P. Biernacki and R. Esman, "RF phase noise performance of a two channel optical down-converting link for microwave phase detection," to appear in IEEE Photonics Technology Letters, vol. 10, no. 4, April, 1998. 